Instituto Internacional de Investigación y Desarrollo Tecnológico Educativo INDTEC, C.A. DOI: https://doi.org/10.29394/Scientific.issn.2542-2987.2018.3.8.3.59-78

OAI-PMH: http://www.indteca.com/ojs/index.php/Revista Scientific/oai

\title{
Mirada Histórica Axiológica del Discurso Formativo del Profesional del Bioanálisis
}

\author{
Autores: Doris Coromoto Pérez De Abreu \\ Universidad de los Andes Núcleo Universitario Rafael Rangel, NURR \\ doris cpda@hotmail.com \\ Trujillo, Venezuela \\ Carol Del Carmen Terán González \\ Universidad Nacional Experimental "Rafael María Baralt", UNERMB \\ carolteranula@gmail.com \\ Trujillo, Venezuela \\ María Inez Albarracín \\ Universidad Nacional Experimental "Rafael María Baralt", UNERMB \\ elprincipitoysurosa2como1@gmail.com \\ Táchira, Venezuela
}

Resumen

Este estudio buscó aproximarse a la realidad teórico axiológico del discurso formativo del profesional del Bioanálisis desde la praxis social. Para ello, se partió de ciertos apartados y procesos dentro de la investigación que circundaron al presente objetivo: Construir aproximaciones teóricas del discurso formativo desde los componentes axiológicos para los profesionales del Bioanálisis en la praxis social del Hospital Universitario de Valera "Dr. Pedro Emilio Carrillo" (HUVPEC), Valera estado Trujillo. La explicación que requirió la trascendencia de esta investigación tuvo su basamento desde la relación e interacción entre el investigador y lo investigado, donde se establecieron representaciones para construir un modelo teórico a partir de la realidad teórico axiológico del discurso formativo del profesional del Bioanálisis desde la praxis social. En cuanto a la argumentación teórica, se desarrolló como unidad temática desde los componentes axiológicos y el discurso de formación del profesional del Bioanálisis. La hermenéutica, representada por Hans-Georg Gadamer, constituye el aspecto metodológico que encausó los senderos de este estudio. Las reflexiones finales del trabajo se concluyeron que todo cambio comienza con una visión y una decisión de emprender la acción, considerando que en el centro psico-biológico social y trascendental del hombre del siglo XXI se encuentra la diversidad, en cuanto a ideas e idealismos, valores, formas de comportamiento, de ser y de pensar. Esta condición puede ser utilizada como herramienta para encontrar sentido al saber, al tener y al saber hacer.

Palabras clave: discurso; formación; profesional.

Fecha de Recepción: 30-12-2016

Fecha de Aceptación: 11-04-2017 


\title{
Axiological Historical Look of the Educational Speech of the Professional of the Bioanalysis
}

\begin{abstract}
This study sought to approach the axiological theoretical reality of the formative discourse of the professional of Bioanalysis from the social praxis. For this, it was started from certain sections and processes within the research that surrounded the present objective: To construct theoretical approaches to the formative discourse from the axiological components for the professionals of the Bioanalysis in the social praxis of the University Hospital of Valera "Dr. Pedro Emilio Carrillo" (HUVPEC), Valera state Trujillo. The explanation that required the transcendence of this research had its basis from the relationship and interaction between the researcher and the researched, where representations were established to build a theoretical model from the axiological theoretical reality of the formative discourse of the professional of Bioanalysis from the praxis Social. As for theoretical argumentation, it was developed as a thematic unit from the axiological components and the training discourse of the professional of Bioanalysis. The hermeneutics, represented by Hans-Georg Gadamer, constitutes the methodological aspect that led the paths of this study. The final reflections of the work concluded that all change begins with a vision and a decision to take action, considering that in the social and transcendental psycho-biological center of the man of the XXI century is diversity, in terms of ideas and idealisms, values, forms of behavior, of being and of thinking. This condition can be used as a tool to find meaning in knowing, having and knowing how to do.
\end{abstract}

Keywords: speeches; training; professional.

Date Received: 30-12-2016

Date Acceptance: 11-04-2017 


\section{A manera de introducción}

Con el advenimiento de la sociedad del conocimiento, el ser humano se ve involucrado en un contexto de sociedad instruida, en la cual su aprendizaje, constituye el instrumento de defensa y seguridad vital más importante para orientarse en el sistema social en el cual está inmerso. No obstante, es importante señalar que no basta con incrementar los conocimientos para cumplir con una misión social, se requiere concatenarlos a un proceso de sensibilización, transformación y valorización del entorno. Este proceso como interpretación social tiene por postulados básicos y operativos la pertinencia social y la pertinencia ocupacional además de ser concebido como la vía para propiciar cambios a través de un continuo desarrollo del ser social. Desde esta perspectiva, la educación cumple un papel importante en el desarrollo cognoscitivo, intelectual y educacional en el individuo, así como también en la familia y en la sociedad.

El proceso formativo se cumple a través de los distintos niveles educativos, garantizando, con ello, su continuidad a lo largo de la vida del individuo mediante un proceso de educación permanente, siendo esta una modalidad que permite producir y utilizar conocimientos que corresponden a los diferentes momentos del desarrollo físico, biológico, psíquico, cultural, social e histórico, en períodos sucesivos donde cada uno engloba al anterior para crear las condiciones de aptitud, capacidad y competencia que le permitan responder y participar activamente en la transformación de la sociedad en la que vive.

En este contexto, se debe el repensar del bioanálisis del siglo XXI; reconfiguración de sus valores axiológicos con base a las nuevas realidades del profesional, sobre todo en base a su perfil, siendo una exigencia ética para todo profesional en función de la necesidad que tiene de actualizar "continuamente" sus conocimientos, habilidades y destrezas para ponerlas al servicio de los ciudadanos, donde el profesional ha comprendido e 
internalizado la importancia de tener una visión ética humanística e integradora en la salud del ser humano, así como una relación con la comunidad, siendo capaz de reconocer el compromiso social que tiene su profesión y el invaluable aporte que tiene el mismo en la solución de los problemas comunitarios a través de la promoción de la salud y la prevención de las enfermedades.

Para hacer frente a este compromiso, el bioanalista. además del conocimiento teórico, del componente de actividades prácticas y del desarrollo de técnicas que constituyen el eje central de su profesión, requiere de una formación humanística y social; para lograrlo, se necesita un discurso formativo emergente desde la universidad cuyo planteamiento debe sustentarse en el desarrollo de sus valores, comprometido con los programas de promoción de salud, asumiendo de esta manera su rol en la praxis social, mediante su participación activa en el proceso científico, socio cultural y gremial de su profesión.

En este marco de ideas, se considera que la función del bioanalista, como la de otros profesionales de la salud, no puede ser aislada de la estructura social ni del sistema de salud vigente, en consecuencia, la formación de este profesional debe estar dirigida a hacer conciencia, solucionar problemas sociales, tecnológicos, científicos y de salud en la comunidad, logrando así la formación de un nuevo profesional, a través de los valores axiológicos en su praxis social como una vía para adecuar y actualizar los nuevos perfiles laborables como consecuencia de las transformaciones acontecidas en el mundo productivo, la nueva realidad de empleo, el desarrollo científico y tecnológico, su historicidad, social política y socio económico al conocimiento epistemológico e interpretativo del ser.

Siendo estas bases sociopolíticas, los modelos económicos y los esquemas y proyectos educativos existentes permitirán facilitar tanto a los docentes como a los estudiantes del sector salud, una nueva conciencia ciudadana con juicios críticos y estructuras de pensamiento que le permitan 
transformar la información en conocimiento, para el buen ejercicio de su profesión y para comprender el porqué de la crisis del sector salud, el problema de la reconfiguración de sus competencias con base a las realidades del perfil profesional y la necesidad de discursos emergentes desde el deber ser del hombre y con contenidos formativos pertinentes.

Los paradigmas emergen en el mundo de hoy, juegan a mitificar los distintos espacios humanos, espacios que forman parte de la calidad de vida; estos contextos son educación, salud, tecnología, es decir, el universo del conocimiento desde distintas corrientes; ahora bien, para el presente estudio el sector salud será la fuente del enriquecimiento para la investigación. Dentro del área de la salud se manifiestan distintos micro mundos profesionales, pero para el sujeto investigador serán los profesionales del bioanálisis los actores sociales de donde se nutrió la producción a desarrollar.

\section{Historia ético-axiológico que circunda al profesional del bioanálisis}

La naturaleza de la formación profesional ha evolucionado a lo largo de los años en estrecha relación con los cambios sociales, con el papel del trabajo en la sociedad y con la diferenciación de los tipos de trabajo; de ahí que, se requiera de profesionales altamente capacitados, formados para la vida, el trabajo y para enfrentar los vertiginosos cambios de la sociedad; por otro lado, las instituciones educativas, las cuales deben estar en consonancia con esas transformaciones, deben ofrecer un amplio abanico de oportunidades de formación y fortalecimiento, promoviendo los procesos de mejora permanente en la calidad de la formación, con la formulación de los perfiles y las bases curriculares, definiendo las competencias profesionales como estándares a todos aquellos profesionales que, habiendo culminado una carrera universitaria y en ejercicio de su profesión, deseen ampliar o profundizar su área de acción para contribuir con sus conocimientos al desarrollo del país.

Sin embargo, una década después un gran número de profesionales 
egresados del Sistema Educativo Universitario encuentra que la mayoría de

sus capacidades, habilidades y conocimientos le son insuficientes para mantenerse o ascender dentro de las estructuras laborales, o mejor aún, para involucrarse y comprometerse de manera activa con la comunidad. Este repensar nos lleva a la formación de un nuevo profesional, a través de los elementos axiológicos que circundan en los valores como una vía para adecuar y actualizar los nuevos perfiles laborables como consecuencia de las transformaciones acontecidas en el mundo productivo, la nueva realidad de empleo, el desarrollo científico y tecnológico, su historicidad social política y socio económico al conocimiento epistemológico.

Teniendo en cuenta estas premisas, Bermúdez (2006: 37), plantea:

Todos los cambios acelerados en los valores y, por ende, en la sociedad, constituyen un desafío para cualquier ética existente, por eso, se requiere de una nueva forma de hacer ética, y ésta es, precisamente, la bioética como ética de la vida, con su carácter interdisciplinario y su papel en la definición e identificación de los problemas, por su metodología para tratarlos y por los espacios que ofrece para la reflexión y toma de decisiones responsables.

Desde estos enunciados, los profesionales del bioanálisis no son solo una delimitación de conocimientos, sino actores sociales de representación de intereses y valores, resistentes a cambios que puedan afectarlos y por supuesto, así ocurre en el campo de la salud. Lo primero a asumir en el personal de salud son las actitudes y valores, su comprensión de ese mundo y las actitudes del escenario de trabajo, así como las metodologías de intervención.

El bioanalista siempre sale restringido al área analítica, base de la carrera, además de estar formado científicamente y técnicamente para efectuar los análisis de laboratorio destinados al diagnóstico de enfermedades, su prevención y tratamiento, para la protección y recuperación de la salud; 
asimismo, debe integrar los valores éticos con el quehacer comunitario para contribuir como agente de cambio social en la búsqueda del bienestar general, asumiendo un rol participativo dentro del equipo de salud, de manera que, conjuntamente con los demás miembros activos de la comunidad puedan desarrollar los mecanismos necesarios para la transformación de los servicios de salud y perfiles epidemiológicos, convirtiéndose así, en un agente permanente desde la responsabilidad del ser y en el actuar con el otro.

En este marco de ideas, la sociedad demanda de los profesionales una nueva concepción de su desempeño, una conciencia ciudadana que reconozca sus derechos y responsabilidades con carácter participativo, protagónico y corresponsable que se exprese en el ámbito social y en la gestión pública; orientado por un modo de vida que centra su fuerza y empuje hacia el desarrollo del equilibrio social, desde una concepción humanista, ambientalista e integracionista.

Desde esta perspectiva, en Venezuela los profesionales están llamados a convertirse en actores sociales comprometidos desde su ser, desde su accionar axiológico con las comunidades para solucionar colectivamente los problemas. De igual forma, las instituciones educativas están emplazadas a formar profesionales que trasciendan en su quehacer social para formar a un nuevo profesional del siglo XXI que propicie la relación de la teoría con la práctica, desde el ser y el actuar en interacción con el ambiente y en una relación horizontal, dialéctica, de reflexión crítica que les permita convertirse en profesionales transformadores, que les permita ser agentes sociales en pro del otro como entes éticos en el actuar humano. En este nuevo enfoque, el profesional del bioanálisis desde los componentes axiológicos como actor social está llamado a asumir un rol protagónico en los cambios que se requieren bajo esta nueva concepción biopsicosocial, axiológicos y espiritual del individuo; a través de valores como el amor comunitario, respeto, responsabilidad y armonía con el otro, la promoción de la salud, prevención de 
la enfermedad, eficiencia en el servicio, entre otros.

Figura 1. Representación mental: Problemática asumida.

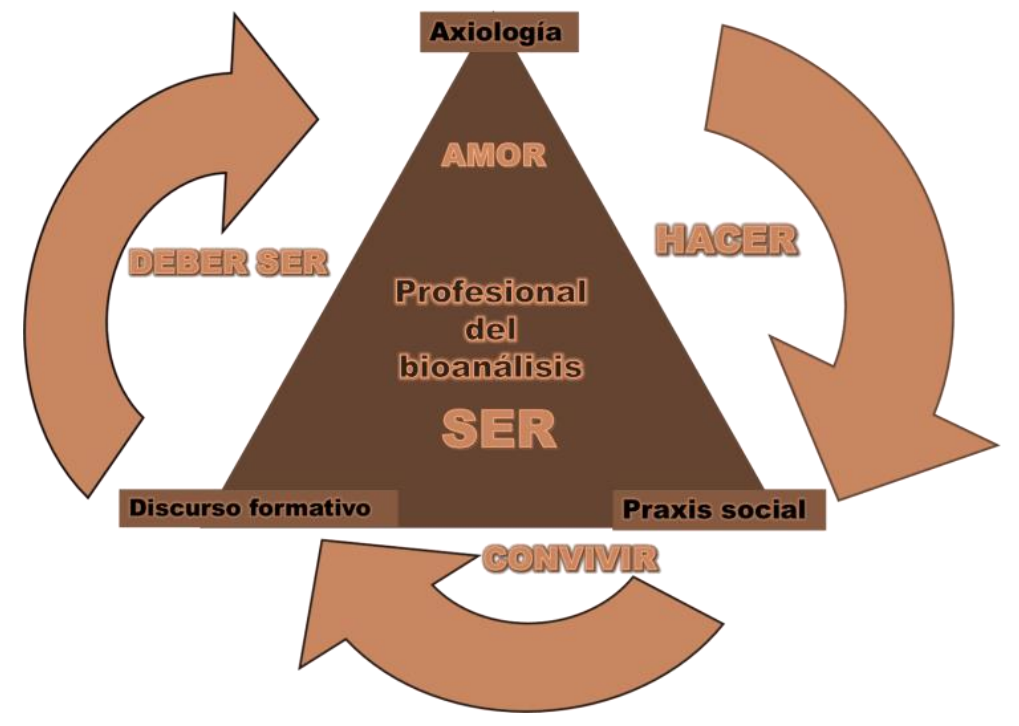

Fuente: Las Autoras (2016).

\subsection{Discurso formativo en el profesional del bioanálisis}

La transformación y la modernidad de los métodos de estudio en la formación de los profesionales universitarios es labor esencial que le concierne a las universidades. Con la llegada del siglo XXI y el avance científico técnico que le precedió y le conduce requiere de profundas reflexiones. Los institutos universitarios son el lugar en el que se experimenta el conjunto de saberes que deberá permitir al futuro profesional iniciarse en el ejercicio de una función con eficacia, así como avanzar en los conocimientos adecuados de la esfera del saber, de modo que consiga iniciarse en la investigación o en la especialización y profundización en su campo de actuación.

Por su parte, Martínez (2006): sustenta que una de las funciones de la formación universitaria es de carácter ético y que no puede entenderse una formación universitaria de calidad que no incorpore de forma sistemática y rigurosa situaciones de aprendizaje ético y de formación ciudadana. Considera 
que la universidad, en el contexto sociocultural actual, es un espacio óptimo de aprendizaje, no sólo de carácter profesional y cultural en su sentido más amplio, sino también de carácter humano y por lo tanto, de carácter ético y moral. Desde estas premisas, se muestra significativamente la importancia que tiene la Declaración Mundial sobre la Educación Superior en el Siglo XXI: Visión y Acción, promulgada durante la Conferencia Mundial sobre la Educación Superior (UNESCO, 1998), donde se refiere en los Artículos 2, 5 y 9 , al papel que ha de jugar la ética, la investigación y las humanidades en todas las disciplinas en una nueva visión y un nuevo modelo de enseñanza superior.

Por otro lado, las instituciones de educación superior, poseen en su discurso un compromiso institucional que busca proveer a los estudiantes un conjunto de particularidades intelectuales y morales, que le consientan interactuar con los demás, incorporando la ética y los valores, para que coexistan competentes de desempeñarse, enfrentar contextos complejos de la vida cotidiana, de modo que puedan ofrecer un servicio profesional de calidad a la sociedad. Desde este punto de vista, Savater (2005:12), sostiene que:

Lo más importante de la educación y por ende, del enseñar, radica no propiamente en transmitir información, sino en aportarle al estudiante la formación de actitudes y valores para la construcción ética del conocimiento, y construirse simultáneamente como personas. La educación es el más humano y humanizador de todos los empeños. Se nace humano, pero eso no basta: tenemos también que llegar a serlo, los humanos nacemos para la humanidad. Hay que nacer para ser humano, pero solo llegamos a serlo plenamente cuando los demás nos contagian su humanidad.

\subsection{Hacia una aproximación del discurso formativo del profesional}

La acción formativa crítica implica un lenguaje innovador desde la pedagogía, que no figura solamente el situar saberes disciplinarios sino también gnosis política y aptitud transformativa de la convivencia social. Desde 
la mirada freiriana la comunicación educacional sería la manera como un sujeto muestra en su relación con el otro, el conocimiento de ser sí mismo, al mismo tiempo que ser conciencia y voluntad de cambiar.

Por ello el hombre convive, lo cual apunta a su disposición en el mundo (saber y pensar). No obstante, su existir deviene en ser, lo cual presume quehacer de apropiación de su existir en el mundo (del espacio de lo aleatorio, pasando en lo explícito/indefinido del ser en el cosmos). Esta coexistencia, trazada como necesidad, práctica y fortaleza, se enuncia en la analogía del sujeto con los otros y lo otro. Este personifica el primer enunciado de situarse en el universo. De esta forma, la propia presencia del sujeto está compuesta del otro y lo otro. Ser y estar, implican, esencialmente, ser y estar en y con el mundo, de allí que la conciencia es, a la vez, personal e histórico -social. Por lo tanto, para Zemelman (1998:32), su planteamiento de que:

La realidad constituye un horizonte de posibilidades, en tanto es historia, actualidad y construcción; es transformación de la historia en experiencia y apertura a lo inédito, entonces, el mundo de la vida sería la actualidad del hombre en tanto conciencia de sí, de la trayectoria de su vida y necesidad de horizontes, esta relación entre cierre y apertura, entre conciencia y voluntad permite la instalación del hombre en el mundo y potencia su aventura, su apropiación, hacia otras realidades, espacios, mundos, lenguajes.

Entonces se puede decir, que la reconstrucción de este discurso cimienta la razón y la práctica del sujeto en las formas cómo se adapta y representa el contexto, ya sea, en tanto objetiva ("realidades tangibles"), como contexto potencial (abierta a las posibilidades) o como perspectivas (complejidad e innovación). A lo que se refiere un sujeto situado, ubicado, que está en el mundo y siendo en ese estar. No se refiere de tener por sabido y dado un mundo de la vida, sino que, para poder objetivarlo, hay que insistente volver a él y refigurarlo (desde sus elementos volitivos, afectivos axiológicos y cognitivos), develarlo para trascender sus propios límites y pasar de lo 
conocido a lo desconocido, desde la realidad práctica, a tal razonamiento Van

Dijk (2001:125), plantea:

El lenguaje y el discurso son fundamentales y constitutivos de lo humano y de la sociedad, y una teoría multidisciplinaria del discurso puede llevarnos bien lejos en la construcción de los puentes o sistemas de traducción. Pero no a través de una reducción al discurso de todos los fenómenos humanos y sociales, sino a través de la construcción de una red teórica en la cual analicemos el discurso en términos de sus estructuras y estrategias cognitivas, lingüísticas, semióticas, interactivas, grupales, institucionales, históricas y culturales. En este sentido, un estudio multidisciplinario del discurso es una manera excelente de relacionar e integrar las ciencias humanas y sociales.

Partiendo de esa relación de ser y hacer en relación con el otro el discurso de formación de profesionales que van a intervenir y trabajar con personas en distintos ámbitos de la vida deben desarrollar y ocuparse de un conjunto de destrezas, recursos y capacidades.

\subsection{Categorías teóricas de la investigación}

Figura 2. Representación mental: Categorías teóricas de la investigación.

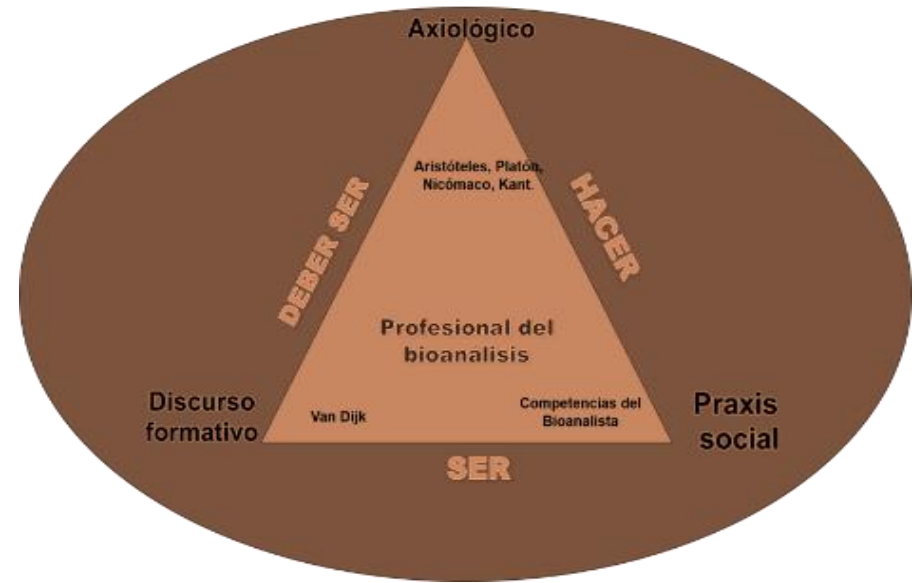

Fuente: Las Autoras (2016). 


\section{Plano Metodológico}

La investigación está enmarcada en el paradigma cualitativo dentro del enfoque interpretativo recursivo, teniendo como método la hermenéutica, Ricoeur citado por Sandoval (1996: 67), expresa que la hermenéutica se define como "la teoría de las reglas que gobiernan una exégesis, es decir, una interpretación de un texto particular o colección de signos susceptible de ser considerada como un texto".

Figura 3. Representación mental: Planos de investigación.

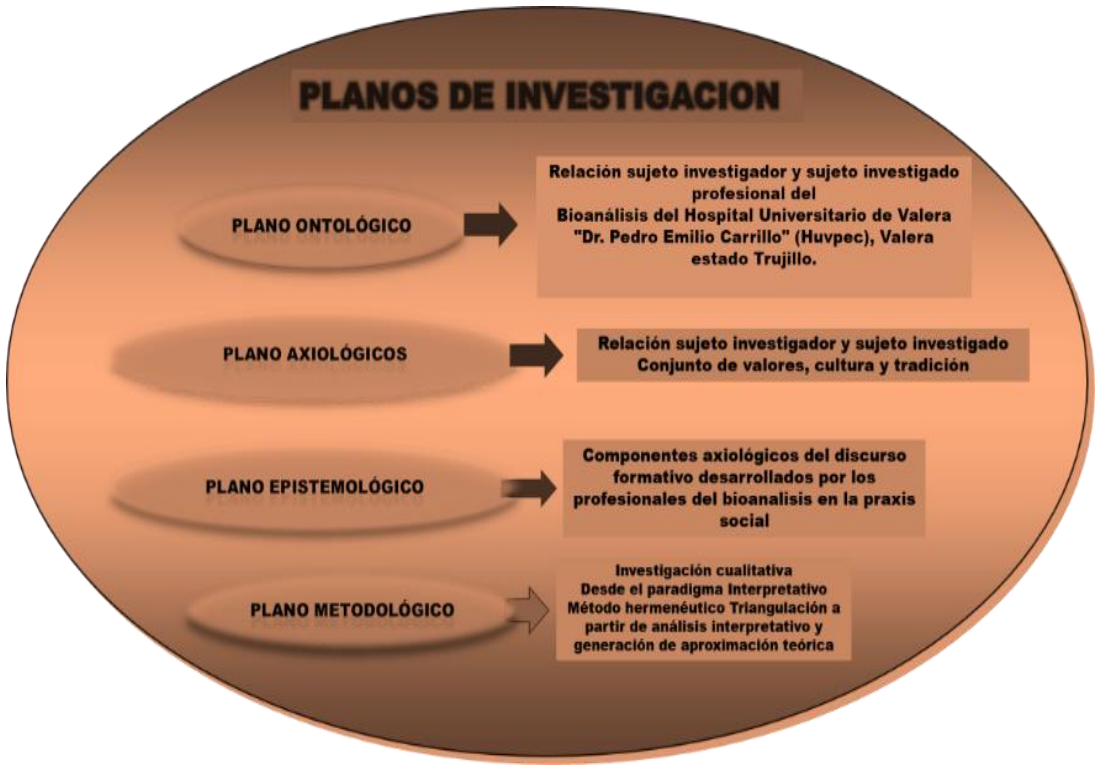

Fuente: Las Autoras (2016).

\subsection{Acercamiento al método}

La hermenéutica como método de investigación propone que la experiencia vivida es esencialmente un proceso interpretativo y se puede realizar desde un horizonte histórico. Van Manen (1997): introduce el enfoque hermenéutico fenomenológico; vincula la fenomenología, hermenéutica y semiótica. La investigación se plantea como un proceso de reflexión textual que contribuye a la comprensión de la acción práctica. La metodología hermenéutica se lleva a cabo exponiendo a la crítica la construcción de cada 
una de las partes involucradas y proporcionando la oportunidad para la revisión de las nuevas construcciones.

El criterio pragmático para esta metodología es que ella conduce, sucesivamente, a una mejor comprensión; esto es, a darle sentido o significado a las interacciones en las cuales uno usualmente está comprometido con otros (...) Hay que establecer un proceso repetitivo en relación con las construcciones existentes (a las que se les dio sentido) para analizarlas y hacer de sus elementos algo sencillo y comunicable para otros. (pág. 35)

La metodología para abordar este proceso de investigación recurre a la hermenéutica de los discursos por los actores sociales: bioanalista que participan en la experiencia. Se requiere comprender las vivencias, las formas en que los actores del proceso experimentan su mundo, qué es lo más significativo para ellos y cómo construyen estos sentidos. Comprensión, interpretación es un proceso conjunto tratando de descifrar los significados y el sentido de la experiencia, a fin de acercarse al proceso en un "círculo hermenéutico", una acción interpretativa como posibilidad de "Hacer hablar" el conocimiento a través de la observación y la reflexión. "El conocimiento tiene la posibilidad de ser "historizado", de hacerse contemporáneo, significado y dar sentido a la experiencia, a fin de acercarse al proceso el investigador que interpreta (el hermeneuta)". (Martínez, 2007:75).

Aquí se pretende, interpretar desde la actuación social, cómo se construye ese mundo simbólico; construcción de sentidos sociales desde los actores del proceso formativo. La descripción representa un primer nivel conceptual con relación al conocimiento de la práctica, se alude a un proceso de reflexión que pretende ordenar u organizar lo que ha sido la marcha de los procesos, buscando en tal dinámica las dimensiones que puedan explicar el teorización más amplio y profundo, contribuyendo a convertirlo en una herramienta para entender y transformar la realidad social. 
Se intenta dilucidar el sentido o el significado que el proceso ha tenido para los actores participantes de creación de conocimientos a partir de nuestra experiencia, de intervención en una realidad social como un primer nivel de teorización sobre la práctica. Representa una articulación entre la teoría y la práctica. Apunta a mejorar la práctica y aspira a enriquecer, confrontar y modificar el conocimiento teórico existente, contribuyendo a convertirlo en una herramienta para entender y transformar la realidad social.

Se pretende entrar en el interior de la dinámica de la experiencia, en su proceso complejo, identificando las contradicciones desde su propia lógica, extrayendo enseñanzas que puedan aportar al enriquecimiento, tanto de la práctica como de la teoría. A partir de textos de observaciones y entrevistas a profundidad de quienes conocen y han vivenciado el proceso e interpretan la realidad; se intenta recuperar lo que saben de su práctica cotidiana, buscando encontrar lo más importante, lo más significativo, descubriendo acontecimientos y dimensiones subjetivas de las personas como: creencias, pensamientos, valores, vivencias, etc.

Esta información resulta fundamental para comprender su propia visión del mundo. Se asume como premisa, lo que miran con los sentidos los actores que las promueven y los resultados de acuerdo con los propósitos que ellos mismos establecieron. Esto permite comprender y mejorar la práctica desde la reflexión y la comprensión del sentido, con una articulación estrecha entre la teoría y la práctica, como una especie particular de creación participativa de conocimientos teórico-prácticos, desde y para la acción de transformación.

Este tipo de metodología está enraizada en la tradición comprensiva e interpretativa de la investigación social; no busca establecer relaciones causales para explicar el fenómeno, busca comprender los aspectos constitutivos de una realidad social; se realiza desde la interacción productora de sentidos para abrir alternativas a mundos posibles. Lo que guía la investigación es el proceso de análisis e interpretación colectiva y construcción 
del sentido de la experiencia, descubrir la estructura compleja de relaciones que conforman la realidad humana, curso de la experiencia realizada.

En el campo metodológico concreto de esta investigación nos encontramos en la necesidad de identificar una los discursos formativos desde lo axiológico que nos permitirá acercarnos a la significatividad del proceso, intentando ubicar las diferentes perspectivas de los actores comprometidos. $Y$ así fue como nos adentramos en las fuentes: valoraciones de los profesionales del bioanálisis y su praxis social; para luego, con este cúmulo de información abordar la interpretación; aquí se tratará de captar el sentido de la experiencia; desde las categorías significativas fuimos ubicando los relatos, los núcleos básicos, hasta descubrir el proceso vivido.

Figura 4. Representación mental: Constructo metodológico.

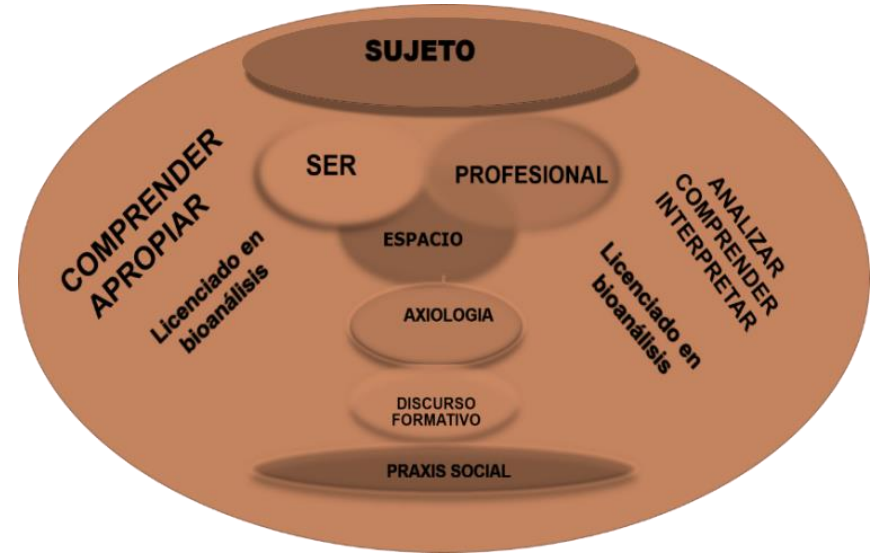

Fuente: Las Autoras (2016).

\section{Reflexiones Finales}

El desarrollo social y económico exige que los sistemas educativos ofrezcan nuevas habilidades y competencias, que les permitan beneficiarse de las nuevas formas emergentes de socialización y contribuyan activamente al desarrollo preventivo de la salud, bajo un sistema cuya principal baza es el conocimiento, adaptable al cambio que pueda construir y desconstruir su aprendizaje, de modo que pueda asumir las innovaciones que se nos presenta 
a diario y así innovarnos de manera de redefinir nuestra condición actual, para lograr la redefinición de las competencias de los profesionales en este caso el Bioanalista del siglo XXI.

Al respecto, Eileen (2003): relaciona las competencias con el conocimiento, los recursos, acceso, tecnología y la habilidad para utilizar este conocimiento como la base, para proveer servicios de alta calidad en cuanto a los servicios informativos y así las competencias personales representan un grupo de actitudes, habilidades y valores que permiten a los profesionales trabajar efectivamente y contribuir positivamente con las organizaciones y su acción social de la misma demostrando su valor de sus contribuciones en un ambiente de constante cambio. Todo cambio comienza con una visión y una decisión de emprender la acción, considerando que en el centro psicobiológico social y trascendental del hombre del siglo XXI se encuentra la diversidad, en cuanto a ideas e idealismos, valores, formas de comportamiento, de ser y de pensar. Esta condición puede ser utilizada como herramienta para encontrar sentido al saber, al tener y al saber hacer. Para que, a través de este sentido, el ser humano llegue a su realización plena y construyan su felicidad en la posesión libre de su ser bondadoso único y verdadero como debe ser.

\section{Referencias}

Bermúdez, C. (2006). Necesidad de la Bioética en la Educación Superior. Acta Bioética. 12(1), 35-40. Recuperado de:

http://dx.doi.org/10.4067/S1726-569X2006000100005

Eileen, B. (2003). Competencias sociales de estudiantes. Barcelona, España: Learson.

UNESCO (1998). La educación superior en el siglo XXI: Visión y acción.

Conferencia Mundial sobre la Educación Superior. Recuperado de: http://www.unesco.org/education/educprog/wche/declaration spa.htm 
Martínez, M. (2007). Comportamiento Humano. Nuevos Métodos de Investigación. México: Editorial Trillas.

Martínez, M. (2006). Formación para la ciudadanía y educación superior. Revista Iberoamericana de Educación, № 42, págs. 85-102. Recuperado de: http://rieoei.org/rie42a05.pdf

Savater, F. (2005). El valor de educar. Barcelona: Ariel S.A.

Sandoval, C. (1996). Investigación cualitativa. México: ARFO Editores.

Van Dijk, T. (2001). El análisis crítico del discurso y el pensamiento social. Athenea Digital, núm. 1: 18-24. Recuperado de: http://www.raco.cat/index.php/Athenea/article/viewFile/34083/33922

Van Manen, M. (1997). Investigación Educativa y Experiencia vivida. Ciencia humana para una pedagogía de la acción y de la sensibilidad. Barcelona, España: 2003 Idea Books, S.A. de la traducción y la edición en lengua castellana, ISBN: 84-B236-283-6. Recuperado de: https://es.scribd.com/doc/172841140/Libro-Investigacion-educativa-yexperiencia-de-vida-Van-Manen

Zemelman, H. (1998). Sujeto: existencia y potencia. Barcelona, España: Editorial Anthropos. 


\section{Doris Coromoto Pérez de Abreu}

e-mail: doris cpda@hotmail.com

Nacida en la ciudad de Valera, Estado Trujillo, Venezuela.

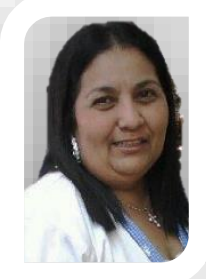

Doctorante en Educación Universidad Rafael María Baralt, Maestría en Docencia para la Educación Superior en la misma universidad, Licenciada en Bioanálisis, ULA. Investigadora en el área de docente, perteneciente a la Línea de investigación Memoria, Educación y Discursos emergentes de la UNERMB. Profesional del bioanálisis con 16 años de experiencia. 


\section{Carol Del Carmen Terán González}

e-mail: carolteranula@gmail.com

Nacida en la ciudad de Valera, Estado Trujillo, Venezuela.

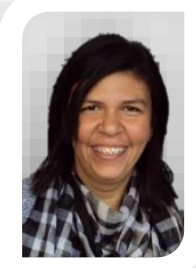

Curso estudios de Doctorado en Educación en la

Universidad Rafael María Baralt, posee Maestría en

Docencia para la Educación Superior en la misma universidad, Maestría en Literatura Latinoamericana en la Universidad de los Andes y Licenciatura en Educación Mención Castellano y Literatura ULA-NURR investigadora del Centro de investigaciones literarias y lingüísticas Mario Briceño Iragorry. (CILL) ULANURR, Jefe de la unidad de investigación y proyecto de la Casa de Historia Trujillo, articulista e investigadora con 14 años de servicio en el área de educación y literatura. Ponente en eventos nacionales e internacionales. Docente invitada en pregrado y Postgrado, ULA, UNERMB, UNESR. Coordinadora de la Línea de investigación Memoria, Educación y Discursos emergentes (UNERMB), miembro de la Línea de investigaciones Ciudadanía, hermenéutica y proyectos sociales. (UNERS). Ha realizado artículos en distintas revistas arbitradas del país entre ella Sapiencia, Cifra Nueva, Revista de Cultura Centro Nacional de Historia, Revista de Ingeniería UVM, entre otras. 


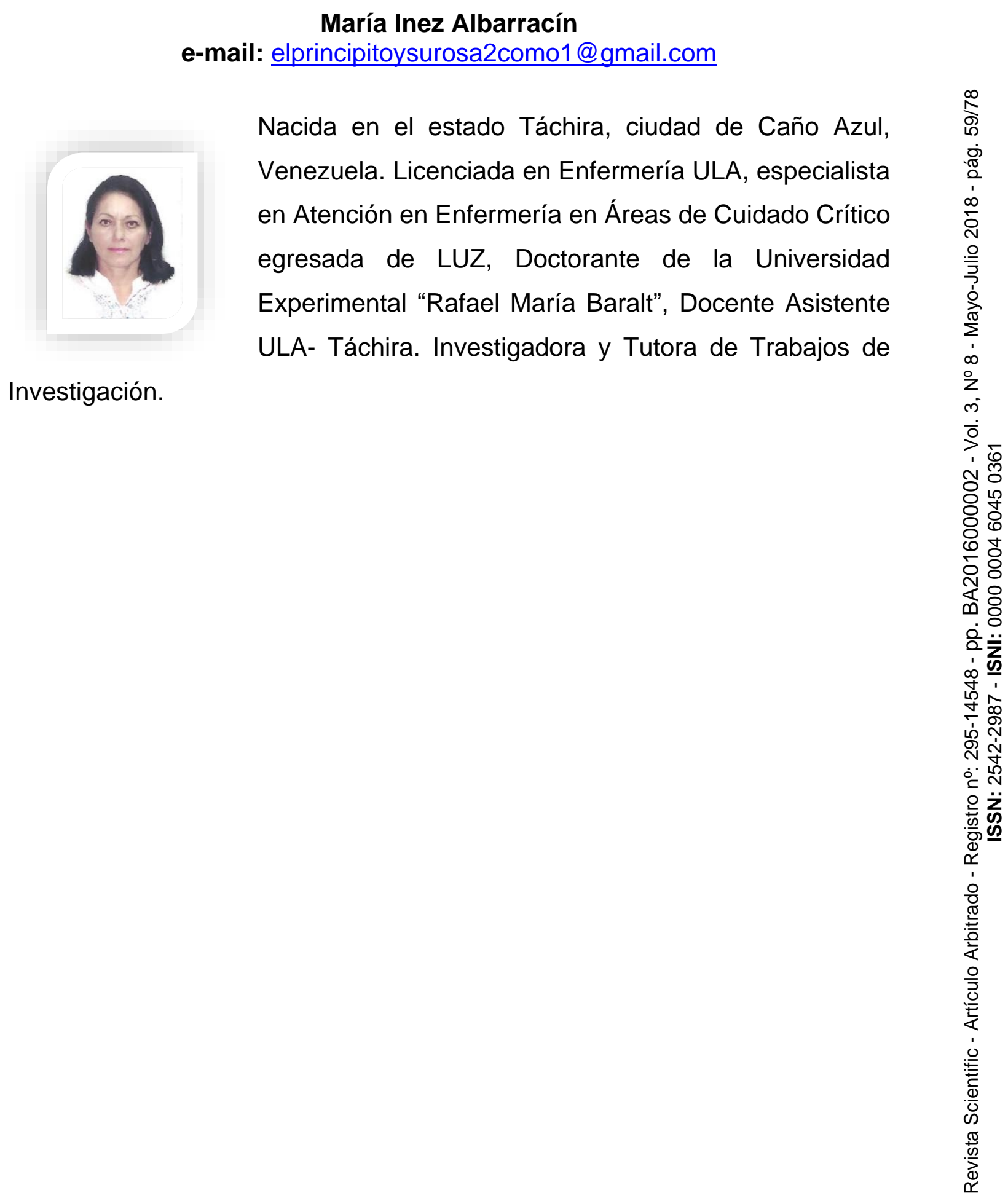

El contenido de este manuscrito se difunde bajo una Licencia de Creative Commons ReconocimientoNoComercial-Compartirlgual 4.0 Internacional 